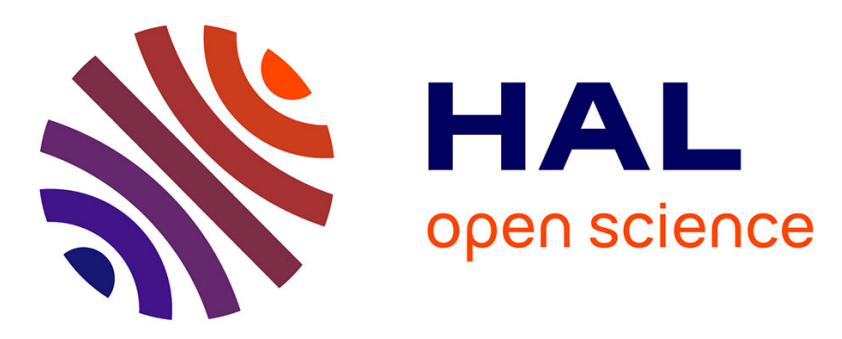

\title{
Singlet exciton fission via an intermolecular charge transfer state in coevaporated pentacene-perfluoropentacene thin films
}

Vincent O Kim, Katharina Broch, Valentina Belova, Y S Chen, Alexander

Gerlach, Frank Schreiber, Hiroyuki Tamura, Raffaele Guido Della Valle, Gabriele d'Avino, Ingo Salzmann, et al.

\section{To cite this version:}

Vincent O Kim, Katharina Broch, Valentina Belova, Y S Chen, Alexander Gerlach, et al.. Singlet exciton fission via an intermolecular charge transfer state in coevaporated pentacene-perfluoropentacene thin films. Journal of Chemical Physics, 2019, 151 (16), pp.164706. 10.1063/1.5130400 . hal03011481

\author{
HAL Id: hal-03011481 \\ https://hal.science/hal-03011481
}

Submitted on 18 Nov 2020

HAL is a multi-disciplinary open access archive for the deposit and dissemination of scientific research documents, whether they are published or not. The documents may come from teaching and research institutions in France or abroad, or from public or private research centers.
L'archive ouverte pluridisciplinaire HAL, est destinée au dépôt et à la diffusion de documents scientifiques de niveau recherche, publiés ou non, émanant des établissements d'enseignement et de recherche français ou étrangers, des laboratoires publics ou privés. 


\section{Singlet exciton fission via an intermolecular charge transfer state in coevaporated pentacene-perfluoropentacene thin films}

Cite as: J. Chem. Phys. 151, 164706 (2019); https://doi.org/10.1063/1.5130400

Submitted: 03 October 2019 . Accepted: 09 October 2019 . Published Online: 30 October 2019

Vincent O. Kim, Katharina Broch (D), Valentina Belova, Y. S. Chen, Alexander Gerlach, Frank Schreiber, Hiroyuki Tamura, Raffaele Guido Della Valle (D), Gabriele D’Avino, Ingo Salzmann (D), David Beljonne (D), Akshay Rao, and Richard Friend

\section{COLLECTIONS}

Paper published as part of the special topic on Singlet Fission

Note: This paper is part of the JCP Special Collection on Singlet Fission.
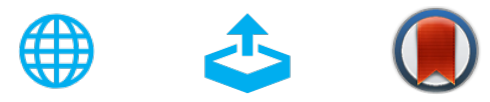

\section{ARTICLES YOU MAY BE INTERESTED IN}

Vibrational probe of the origin of singlet exciton fission in TIPS-pentacene solutions The Journal of Chemical Physics 151, 154701 (2019); https://doi.org/10.1063/1.5116586

Fluctuating exchange interactions enable quintet multiexciton formation in singlet fission The Journal of Chemical Physics 151, 164104 (2019); https://doi.org/10.1063/1.5115816

Synthesis and optical properties of colloidal $\mathrm{Cs}_{2} \mathrm{AgSb}_{7-x} \mathrm{Bi}_{x} \mathrm{Cl}_{6}$ double perovskite nanocrystals

The Journal of Chemical Physics 151, 161101 (2019); https://doi.org/10.1063/1.5127971

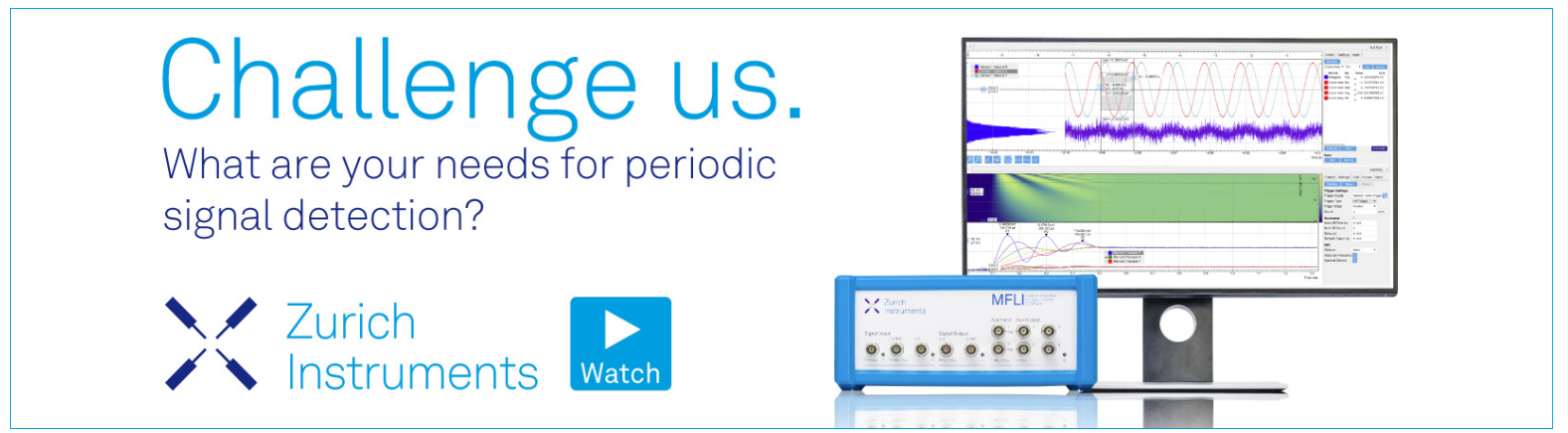




\title{
Singlet exciton fission via an intermolecular charge transfer state in coevaporated pentacene-perfluoropentacene thin films
}

Cite as: J. Chem. Phys. 151, 164706 (2019); doi: 10.1063/1.5130400

Submitted: 3 October 2019 - Accepted: 9 October 2019 •

Published Online: 30 October 2019

\author{
Vincent O. Kim, ${ }^{1, a)}$ Katharina Broch, ${ }^{2, a)}$ (D) Valentina Belova, ${ }^{3, a)}$ Y. S. Chen, ${ }^{1, a)}$ Alexander Gerlach, \\ Frank Schreiber, ${ }^{3, a)}$ Hiroyuki Tamura, ${ }^{4, a)}$ Raffaele Guido Della Valle, ${ }^{5, a)}$ (D) Gabriele D'Avino, ${ }^{6, a)}$ \\ Ingo Salzmann, ${ }^{7, a)}$ (D) David Beljonne, ${ }^{8, b)}$ (D) Akshay Rao, ${ }^{1, a)}$ and Richard Friend ${ }^{1, a)}$
}

\begin{abstract}
AFFILIATIONS
${ }^{1}$ Cavendish Laboratory, Department of Physics, University of Cambridge, Cambridge CB3 OHE, United Kingdom

${ }^{2}$ Fritz Haber Institute of the Max Planck Society, Department of Physical Chemistry, Faradayweg, 4-614195 Berlin, Germany

${ }^{3}$ Eberhard-Karls Universität Tübingen, Institut für Angewandte Physik, Auf der Morgenstelle 10, 72076 Tübingen, Germany

"Department of Chemical System Engineering, The University of Tokyo, Tokyo 113-8656, Japan

${ }^{5}$ Dipartimento di Chimica Industriale "Toso Montanari", Università di Bologna and INSTM-UdR Bologna,

Viale Risorgimento 4, 40136 Bologna, Italy

${ }^{6}$ Institut Néel, CNRS and Grenoble Alpes University, F-38042 Grenoble, France

${ }^{7}$ Department of Physics, Department of Chemistry and Biochemistry, Centre for Research in Molecular Modeling (CERMM), Centre for NanoScience Research (CeNSR), Concordia University, 7141 Sherbrooke St. West, Montreal, Quebec H4B 1R6, Canada ${ }^{8}$ Laboratory for Chemistry of Novel Materials, Department of Chemistry, Université de Mons, Place du Parc 20,

7000 Mons, Belgium
\end{abstract}

\begin{abstract}
Note: This paper is part of the JCP Special Collection on Singlet Fission.
a)kimv74@mail.wlu.edu; katharina-anna.broch@uni-tuebingen.de; vbelova@icmab.es; ysc34@cam.ac.uk; alexander.gerlach@uni-tuebingen.de; frank.schreiber@uni-tuebingen.de; tamura.hiroyuki@gmail.com; raffaele.dellavalle@unibo.it; gabriele.davino@gmail.com; ingo.salzmann@concordia.ca; ar525@cam.ac.uk; andrhf10@cam.ac.uk

${ }^{\text {b) }}$ Author to whom correspondence should be addressed: David.BELJONNE@umons.ac.be
\end{abstract}

\begin{abstract}
Singlet exciton fission is a spin-allowed process in organic semiconductors by which one absorbed photon generates two triplet excitons. Theory predicts that singlet fission is mediated by intermolecular charge-transfer states in solid-state materials with appropriate singlettriplet energy spacing, but direct evidence for the involvement of such states in the process has not been provided yet. Here, we report on the observation of subpicosecond singlet fission in mixed films of pentacene and perfluoropentacene. By combining transient spectroscopy measurements to nonadiabatic quantum-dynamics simulations, we show that direct excitation in the charge-transfer absorption band of the mixed films leads to the formation of triplet excitons, unambiguously proving that they act as intermediate states in the fission process.
\end{abstract}

Published under license by AIP Publishing. https://doi.org/10.1063/1.5130400

Singlet exciton fission (SF) is a spin-allowed process in organic semiconductors by which one singlet exciton $\left(S_{1}\right)$ splits to form two triplet excitons $\left(T_{1}\right){ }^{1}$ First reported in the $1960 \mathrm{~s}$, ${ }^{2,}$

it has recently been scrutinized for its potential to enhance the efficiency of photovoltaic (PV) cells. This phenomenon could allow devices to surpass the Shockley-Queisser limit and raise the maximum theoretical photovoltaic power conversion efficiency from $33.7 \%$ to $44.4 \%$. ${ }^{4}$ Singlet fission has been observed in 
working devices, ${ }^{5-8}$ but detailed mechanistic aspects are still being debated.

There are two overarching mechanisms for singlet fission: (i) a two-electron process involving a direct coupling between the ${ }^{1}\left(\mathrm{~S}_{1} \mathrm{~S}_{0}\right)$ state and a multiexciton ${ }^{1}\left(\mathrm{~T}_{1} \mathrm{~T}_{1}\right)$ state and (ii) a combination of two one-electron transfer events involving intermediate charge-transfer (CT) states. ${ }^{1}$ These may either be transiently populated or act as virtual states in a superexchangelike mechanism. However, the interplay between the various states during SF has been demonstrated to be more complex. ${ }^{9-17}$ While there is much debate surrounding the theory of CT-mediated singlet fission, experimental proofs providing direct evidence for the role of CT states are scarce. Walker et al. found that an excited TIPS-pentacene molecule interacts with a ground state TIPS-pentacene molecule to form a transient bound excimer intermediate during singlet fission in solution. ${ }^{18}$ Busby et al. were able to design molecules and polymers with covalently bound, strongly coupled donor and acceptor units that exhibit intramolecular singlet fission mediated by CT states. ${ }^{19}$ Controlling the CT state energy in terrylene diimide dimers has been shown to either inhibit or promote singlet fission in solution, and that study was the first time a CT intermediate state was directly observed in a solution exhibiting singlet fission. ${ }^{20}$ However, the observation of real CT intermediates during ultrafast fission in the solid state has proved challenging, which makes it difficult to draw definitive conclusions on their role in the fission process.

Here, we address this question by turning our attention to coevaporated blends of pentacene (PEN) and perfluoropentacene (PFP). PFP:PEN films have previously been found to display an (additional with respect to pure PEN and PFP films) intermolecular charge-transfer band peaking at $\sim 1.6 \mathrm{eV}$ on the optical absorption spectrum, i.e., below the onset of absorption of PEN and PFP. ${ }^{21,22}$ We combine transient absorption (TA) spectroscopy to excited-state quantum-dynamics simulations in order to probe the growth and decay of the singlet and triplet state populations in these systems. Our results support a sequential process, whereby the formation of CT states generated either by direct excitation or transiently populated from PEN/PFP singlet excitons is followed by their conversion into triplets.

The samples were prepared on $1 \mathrm{~mm}$-thick fused silica substrates using molecular beam deposition in an ultrahigh vacuum chamber (base pressure $2 \times 10^{-10} \mathrm{mbar}$ ) at a substrate temperature of $300 \mathrm{~K}$, a growth rate of $0.2 \mathrm{~nm} / \mathrm{min}$, and a final film thickness of $100 \mathrm{~nm}$. A water-cooled quartz-crystal microbalance, which was calibrated via X-ray reflectivity measurements, was used to monitor the growth. For the mixed films, the rates of the single components were set prior to the deposition; in total, we prepared four samples with different mixing ratios of PFP and PEN (3:1, 1:1, 1:2, and 1:3). Previous structural characterization has shown that the $1: 1$ blend is intimately mixed, but not a perfect cocrystal. Increasing the ratio of one component leads to the formation of domains of pure materials as well as intimately mixed regions. More details of sample characterization and a discussion of the optical properties of the films are reported in Ref. 21.

Ultrafast (30 fs-resolution) transient absorption measurements were performed in a configuration that resembles the one previously reported ${ }^{23}$ but uses an amplifier incorporating that produced $14.5 \mathrm{~W}$ at $1030 \mathrm{~nm}$ at a repetition rate of $38 \mathrm{kHz}$ (PHAROS, Light
Conversion). This was coupled to a noncollinear optical parametric amplifier (NOPA) $\left[37^{\circ}\right.$ cut $\beta$-barium borate (BBO), type $\mathrm{I}, 5^{\circ}$ external angle] that generated broadband $(25 \mathrm{~nm})$ excitation pulses allowing a time resolution of $30 \mathrm{fs}$ through chirped mirror compression. The pump photon energies ranged from $1.9 \mathrm{eV}$ to $2.3 \mathrm{eV}$ with a fluence of $3.5 \times 10^{14}$ photons/pulse $\mathrm{cm}^{2}$. The transmission through the samples was probed using broadband pulses $(540 \mathrm{~nm}$ $-1000 \mathrm{~nm}$ ) from the focusing part of the $1030 \mathrm{~nm}$ fundamental onto a $3 \mathrm{~mm}$ YAG crystal. We split the probe beam to provide a reference signal from the samples that was not influenced by the excitation pulses. This reference reduces the effects of the fluctuating laser. The pump and probe are overlapped on the sample at an $8.7^{\circ}$ angle, and a chopper wheel running at $500 \mathrm{~Hz}$ blocks the pump so that we can probe transmission with and without the excitation pulse. The probe and reference are then directed into a monochromator and detected using an InGaAs photodiode array camera (Sensors Unlimited/BF Goodrich). We measured the differential transmission $(\Delta \mathrm{T} / \mathrm{T})$ as a function of probe photon energy and temporal delay between the pump and the probe. The delay is controlled by a computer-operated piezoelectric translation stage.

The crystal structure and ground-state absorption of various PFP:PEN crystals are displayed in Figs. 1(a) and 1(b), respectively. In Fig. 1(b), $\varepsilon$ is the imaginary part of the in-plane component of the dielectric function measured with differential reflectance spectroscopy (DRS). PFP:PEN 1:3 shows the strongest absorption in the
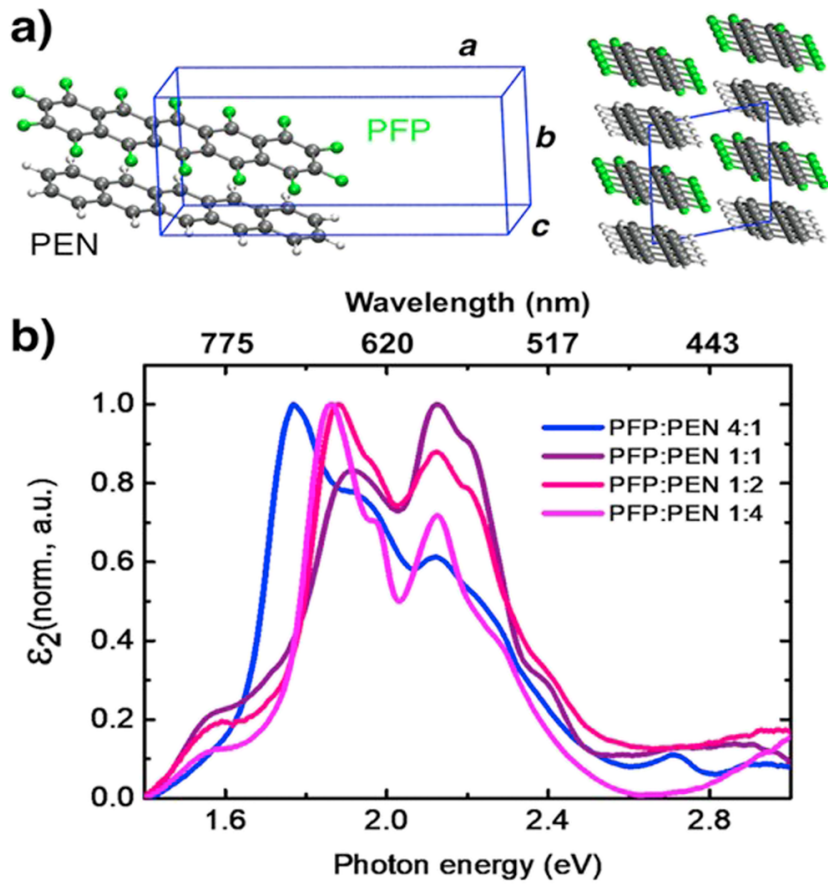

FIG. 1. Crystal structure and ground-state absorbance spectra of coevaporated PFP:PEN films. (a) Unit cell and molecular packing in the PFP:PEN 1:1 crystal. (b) The ground-state absorbance spectra of various blends of PFP:PEN, where $\varepsilon$ is the imaginary part of the in-plane component of the dielectric function measured with differential reflectance spectroscopy (DRS). 
region of the $\mathrm{CT}$ state around $1.6 \mathrm{eV}$, and the $\mathrm{CT}$ state absorption is less than $10 \%$ of the maximum absorption of the materials.

We perform ultrafast (30 fs resolution) TA measurements to track the spectral evolution of pure PEN, pure PFP, and the PFP:PEN thin films (Fig. 2). We use a broadband probe that extends from $650 \mathrm{~nm}$ to $925 \mathrm{~nm}$. Figure 2(a) displays the TA spectra of a pure PEN film that resembles the spectra previously reported, ${ }^{22,24,25}$ where a sub-100 fs time constant was observed for the fission process. The pure PEN spectra exhibit a positive ground-state bleach (GSB) signal centered at $685 \mathrm{~nm}$ that corresponds to the pump excitation depopulating the ground state. The $685 \mathrm{~nm}$ peak is related to the $\mathrm{S}_{0} \rightarrow \mathrm{S}_{1}$ transition. This overlaps with a positive stimulated emission (SE) signal at $720 \mathrm{~nm}$ that can be seen at the earliest times. A negative signal from $720 \mathrm{~nm}$ to $925 \mathrm{~nm}$ is the broad photoinduced absorption (PIA) of triplets. This PIA signal relates to the $\mathrm{T}_{1} \rightarrow \mathrm{T}_{2}$ transition in linear acenes. ${ }^{24}$

The spectra of the pure PFP film [Fig. 2(b)] appears similar to the spectra of the pure PEN film with the former being slightly redshifted. The PFP film exhibits a positive GSB signal centered at $700 \mathrm{~nm}$ that we attribute to the $S_{0} \rightarrow S_{1}$ transition by analogy to the PEN spectra in Fig. 2(a). A positive SE feature occurs in the PFP film at $735 \mathrm{~nm}$. We assign the negative PIA feature around $740 \mathrm{~nm}$ that extends to $925 \mathrm{~nm}$ to a $\mathrm{T}_{1} \rightarrow \mathrm{T}_{2}$ transition, again, by analogy to the similar feature in PEN. Because this system resembles PEN, and triplets are forming within $200 \mathrm{fs}$, i.e., too quickly for intersystem crossing, we conclude that singlet fission is occurring in the pure PFP film, in agreement with the previous work reporting subpicosecond fission rate in this system.

Figure 2(c) displays the TA spectra of the PFP:PEN 1:3 film. The positive feature at $680 \mathrm{~nm}$ is the GSB and resembles the GSB of the pure PEN film rather than the $700 \mathrm{~nm}$-peaked GSB of the pure PFP film, and we attribute this feature at $680 \mathrm{~nm}$ to an $S_{0} \rightarrow S_{1}$ transition in PFP:PEN 1:3. There is a small positive shoulder at $710 \mathrm{~nm}$ that we attribute to the SE of the singlet excitons based on a similar feature in the spectra of both PFP and PEN. However, there is another positive peak at $795 \mathrm{~nm}$ that is not present in the spectra of either the pristine PEN or PFP. Thus, we attribute this feature to the bleaching of the intermolecular CT state between PEN and PFP in the PFP:PEN 1:3 blend, based on the absorption spectra shown in Fig. 1. This feature is superimposed on a broad PIA feature extending from $720 \mathrm{~nm}$ to $925 \mathrm{~nm}$, which we assign to a $T_{1} \rightarrow T_{2}$ transition based on comparison to the PEN and PFP spectra. Therefore, we a) PEN

Photon Energy (eV)

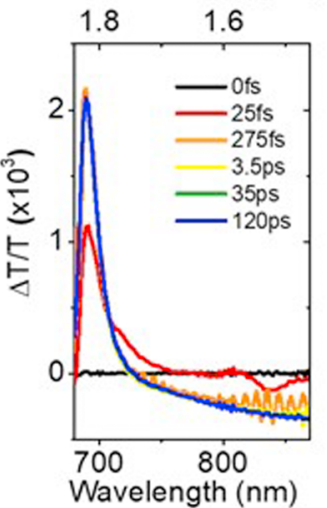

d) PFP:PEN 1:2

Photon Energy (eV)

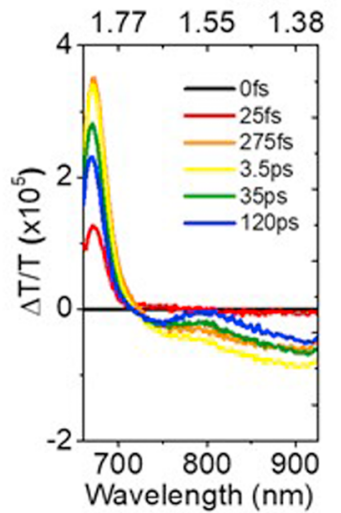

b) PFP
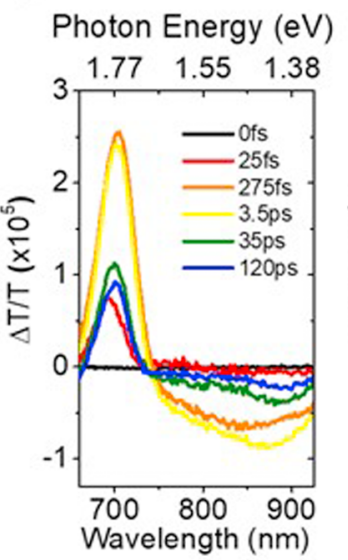

e) PFP:PEN 1:1

Photon Energy (eV)

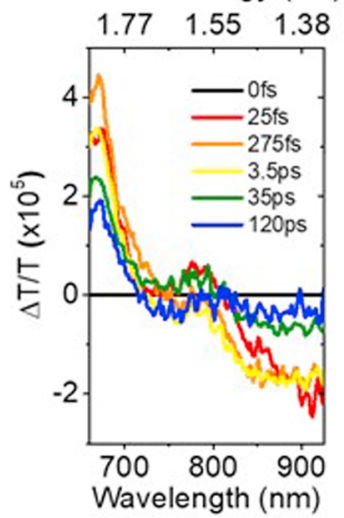

c) PFP:PEN 1:3

Photon Energy (eV)

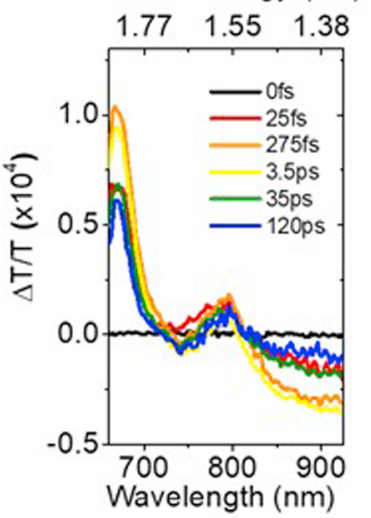

f) PFP:PEN 3:1

Photon Energy (eV)

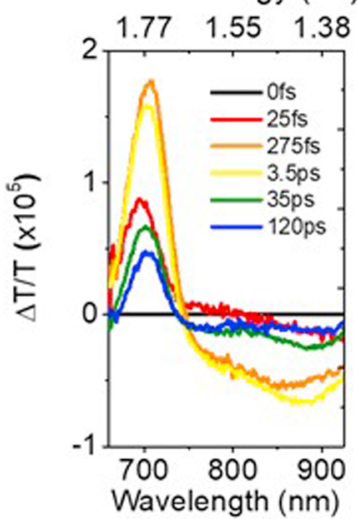

FIG. 2. Transient absorption spectra of pure and mixed films of pentacene (PEN) and perfluoropentacene (PFP) grown on $\mathrm{SiO}_{2}$. Transient spectra of (a) pure PEN, (b) pure PFP, (c) PFP:PEN 1:3, (d) PFP:PEN 1:1, (e) PFP:PEN 2:1, and (f) PFP:PEN 3:1. The mixed blends exhibit an intermolecular charge transfer state centered around $800 \mathrm{~nm}$ that is seen most clearly in the spectra of the PFP:PEN 1:3 blend and nonexistent in the spectra of the pure PFP and pure PEN films. The films were excited at 650 $\mathrm{nm}$ at a fluence of $118 \mu \mathrm{J} / \mathrm{cm}^{2}$ per pulse. 
conclude that singlet fission is also occurring in the PFP:PEN 1:3 film and that an intermolecular CT state is being populated during this process.

We see similar TA spectra from the PFP:PEN 1:2 film [Fig. 2(d)]. There is a positive GSB peak at $680 \mathrm{~nm}$ and a positive SE feature at $710 \mathrm{~nm}$. We attribute a $\mathrm{T}_{1} \rightarrow \mathrm{T}_{2}$ transition to the broad PIA from $720 \mathrm{~nm}$ to $925 \mathrm{~nm}$. While there is no positive feature at $795 \mathrm{~nm}$, there is a dampening of the negative signal there, which suggests that there is an overlapping positive signal from the bleach of an intermolecular CT state. We suspect that the ratio of the CT state signal to the PIA signal in the PFP:PEN 1:2 film is smaller than the ratio of the two signals in the PFP:PEN 1:3 film. However, given the fast rise of the triplet signal and the presence of similar spectral features as in the pure materials and blends, the PFP:PEN 1:2 film also seems to exhibit singlet fission.

The TA spectra from the PFP:PEN 1:1 film [Fig. 2(e)] resemble those of the PFP:PEN 1:2 film and, especially, those of the PFP:PEN 1:3 film. A positive GSB peak occurs at $680 \mathrm{~nm}$, a positive SE signal is observed at $710 \mathrm{~nm}$, and there is a positive signal at $790 \mathrm{~nm}$ that we attribute to the bleaching of an intermolecular CT state. As in the PFP:PEN 1:3 blend, this positive CT feature overlaps with a broad negative PIA feature that extends from $720 \mathrm{~nm}$ to $925 \mathrm{~nm}$, which we also attribute to a $\mathrm{T}_{1} \rightarrow \mathrm{T}_{2}$ transition. The spectra of PFP:PEN 1:1 resembles those of PFP:PEN 1:3 to the extent that we conclude that the PFP:PEN 1:1 film also undergoes singlet fission.

PFP:PEN 3:1, the only mixed film containing more PFP than PEN discussed in this study, has its TA spectra displayed in Fig. 2(f). There is a positive GSB feature centered at $700 \mathrm{~nm}$, which resembles the GSB of pristine PFP rather than the GSB of pristine PEN. Like the other films, we attribute this peak to a $S_{0} \rightarrow S_{1}$ transition. A positive SE signal is present at $730 \mathrm{~nm}$, and there is a broad PIA signal from $730 \mathrm{~nm}$ to $925 \mathrm{~nm}$ that we characterize as a $T_{1} \rightarrow T_{2}$ transition. As in the PFP:PEN 1:2 film, this negative PIA feature is dampened around $800 \mathrm{~nm}$ (and even positive at early times). Therefore, the bleach of an intermolecular CT state probably coincides with the triplet PIA and, consequently, singlet fission occurring in this film.

Thus, the ultrafast TA experiments suggest that singlet fission is occurring in both the pristine and mixed films of PFP and PEN. Furthermore, we directly observe the bleaching of the CT state in the TA spectra of each of the blends. The CT state feature is most prominent in the PFP:PEN 1:3 blend, with the feature in the PFP:PEN 1:1 being the next most prominent. The CT state features in the PFP:PEN 3:1 and PFP:PEN 1:2 appear weaker and are obscured by the triplet PIA. Overall, the bleach of the CT states corresponds to the steadystate absorption measurements in Fig. 1 as well as previous timedependent density functional theory (TD-DFT) calculations. ${ }^{27}$ As we shift attention now to the role of the CT state in singlet fission, we focus on the PFP:PEN 1:3 film because it exhibits the strongest CT signal. We believe that this strong CT feature helps illustrate the role of the CT state in singlet fission.

Figure 3 shows the dynamics of three populations in the spectra of PFP:PEN 1:3 from Fig 2(e). They are kinetic cuts of the spectra representing the stimulated emission of the singlet exciton population on top of the ground state bleach $(700-710 \mathrm{~nm})$, the bleach of the CT state $(780-810 \mathrm{~nm})$, and the PIA of the triplet

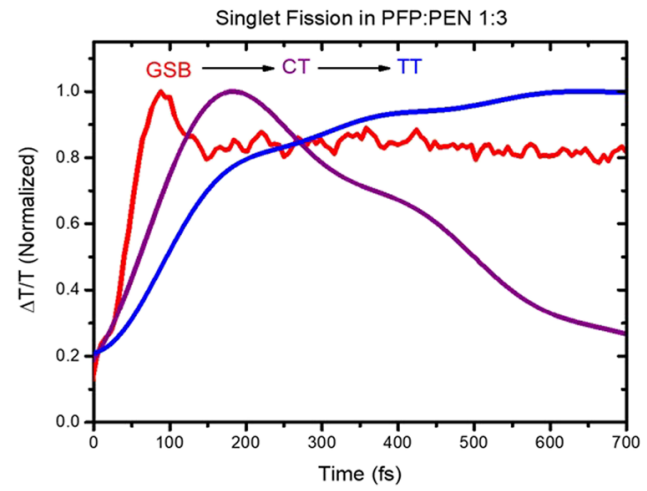

FIG. 3. Dynamics of singlet exciton fission (SF) in a film of PFP:PEN 1:3. Kinetic cuts taken from the spectra in Fig. 2(c). The singlet curve is the stimulated emission of the singlet exciton cut from $700 \mathrm{~nm}$ to $710 \mathrm{~nm}$. The charge transfer state is cut from $780 \mathrm{~nm}$ to $810 \mathrm{~nm}$, and the triplet curve is the photoinduced absorption cut from $900 \mathrm{~nm}$ to $925 \mathrm{~nm}$. A decay of the singlet population coincides with the rise of a CT state population. Then, as the CT state population decays, a triplet population forms at a similar rate $(500 \mathrm{fs})$. This sequence indicates a CT-mediated SF process where the intermolecular CT state between PFP and PEN mediates SF in mixed films of PFP:PEN.

population (900-940 $\mathrm{nm}$ ). We first see that there is a sharp drop in the stimulated emission at early times, corresponding to a loss of singlet excitons. This is concomitant with a rise in the CT state population. We then observe the CT state population decay with a time constant of $500 \mathrm{fs}$, while the triplet population grows with a similar time constant of $600 \mathrm{fs}$. This sequence of events suggests that the CT state acts as an intermediary in singlet fission in PFP:PEN 1:3, and because the CT state is similar in the other blends, it is likely to play a similar role in all of the PFP:PEN blends.

We performed another femtosecond-TA measurement (on a $1 \mathrm{kHz}$ Ti:sapphire laser system, $800 \mathrm{~nm}$ fundamental, $100 \mathrm{fs}$ time resolution) on the same PFP:PEN 1:3 film referenced in Fig. 2(c) with the broadband pump tuned to $800 \mathrm{~nm}$ in order to directly excite the CT state. The pump fluence was set to $9.5 \mathrm{~mJ} / \mathrm{cm}^{2}$ per pulse (at a repetition rate of $1 \mathrm{kHz}$ ). Such a high fluence was needed to obtain the $\Delta \mathrm{T} / \mathrm{T}$ signal because, as shown in Fig. 1(b), the film absorbs very little light at $800 \mathrm{~nm}$. The signal measured scaled linearly suggesting very low two-photon absorption contributions in these measurements. Figure 4 depicts the TA spectrum of the PFP:PEN 1:3 film averaged over the range of 600-800 fs. This spectrum agrees with the spectra when exciting the same PFP:PEN 1:3 film at $650 \mathrm{~nm}$ above the bandgap. Here, the positive GSB feature at $670 \mathrm{~nm}$ resembles the GSB of the pure PEN film more than the $700 \mathrm{~nm}$ GSB peak of the pure PFP film. Again, we attribute this feature at $670 \mathrm{~nm}$ to an $S_{0} \rightarrow S_{1}$ transition in PFP:PEN 1:3. There is a similar positive peak at $785 \mathrm{~nm}$ that is not present in either of the spectra of pristine PEN or PFP. Therefore, we conclude this feature to be the bleaching of the intermolecular CT state between PEN and PFP in the PFP:PEN 1:3 blend. As before, the CT feature overlaps with a broad PIA feature extending from 720 to $925 \mathrm{~nm}$, which corresponds to singlet and triplet excitons based on comparison to the spectra in Figs. 2(a)-2(c). Therefore, we conclude that singlet fission 


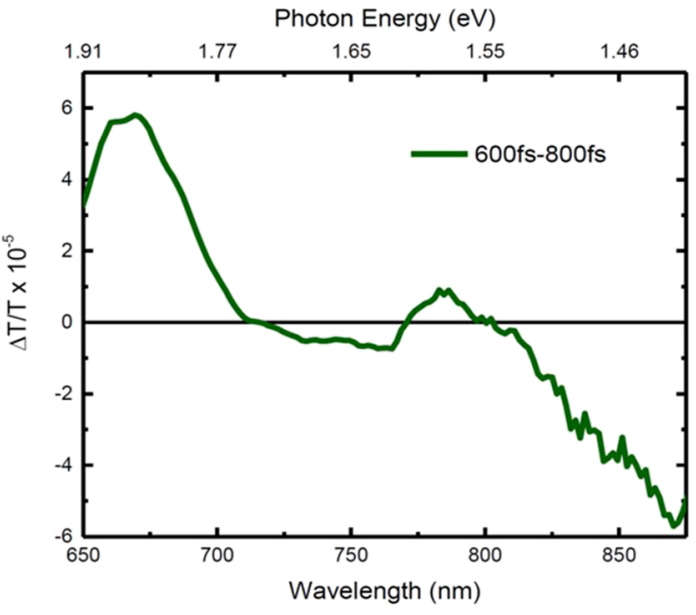

FIG. 4. Transient absorption spectrum of a PFP:PEN 1:3 film when the charge transfer state is directly excited at $800 \mathrm{~nm}$ averaged over the range of $600-800 \mathrm{fs}$. This spectrum resembles the spectra when exciting the same PFP:PEN 1:3 film at $650 \mathrm{~nm}$ above the bandgap. In particular, directly exciting the CT state results in an observable bleaching of the CT state around $780-800 \mathrm{~nm}$.

is also occurring in the PFP:PEN 1:3 film excited via the CT states at $800 \mathrm{~nm}$. This directly provides experimental evidence that the CT state feeds the fission process. We note that the very small signal observed $\left(10^{-5}\right)$ is at the limits of what can be measured using our $1 \mathrm{kHz}$ TA system and impedes a detailed analysis of early time dynamics, which is also contaminated by the presence of a strong coherent artefact.

To complement these data, a computational study has been performed in order to (i) resolve an all-atom crystal structure, (ii) assess the nature and energetics of the relevant electronic excitations, and (iii) model singlet fission dynamics in the mixed PEN:PFP cocrystals. The existence of a PFP:PEN mixed crystal structure with 1:1 stoichiometry has been deduced from XRD and grazing incidence $\mathrm{x}$-ray diffraction (GIXRD) measurements. ${ }^{28-30}$ Although atomic coordinates could not be obtained from experimental data, the GIXRD diffractogram is sufficient to determine the unit cell parameters ${ }^{31,32}$ from the peak positions and to confirm or exclude a given structure by the peak intensities. GIXRD yields a P-1 triclinic unit cell for the PEN:PFP cocrystals (lattice parameters $a=15.7 \AA, b=7.4 \AA, c=7.2 \AA, a=102.8^{\circ}$, $\beta=68.6^{\circ}$, and $\gamma=97.6^{\circ}$ ). The comparison between experimental intensities in GIXRD and calculated values for guess structures from force-field calculations allowed us to conclude that the unit cell comprises one PEN and one PFP cofacial molecule forming an alternating (mixed) stack along the crystallographic $b$ axis [see Fig. 1(a).$^{29}$ PEN and PFP molecules lie at $(0,0,0)$ and $(0,0.5,0)$ positions, respectively, featuring an intermolecular distance $b / 2=3.7 \AA$ that ensures a sizable overlap between their frontier molecular orbitals.

Next, we theoretically characterized the excited-state energetics of the PEN:PFP cocrystals by combining $a b$ initio to charge response $(\mathrm{CR})^{33,34}$ modeling schemes. CR calculations provide an accurate description of the electrostatic and induction interactions in the solid state that can largely affect the energies of charge carriers and CT excitations in molecular crystals. ${ }^{35}$ The contribution from intermolecular interactions to CT states energy is quantified by the polarization energy $\Delta^{\mathrm{CT}}$ that we compute for electron-hole pair excitations with charges strictly localized on molecular units. The lowest-energy ion pair is, as expected, for the configuration with the hole on PEN and the electron on the nearest-neighbor PFP in the 1:1 structure. We have computed the polarization energy for an isolated PEN:PFP dimer $\left(\Delta^{\mathrm{CT}}=-1.85 \mathrm{eV}\right)$ and for the same dimer embedded in the crystal $\left(\Delta^{\mathrm{CT}}=-1.98 \mathrm{eV}\right)$. The CT energies of the isolated and embedded complex hence differ by only $\sim 0.1 \mathrm{eV}$. This result is specific to the alternating PEN:PFP molecular packing and is due to the compensation of electrostatic embedding and dielectric screening in the solid (see the supplementary material for details). The PEN:PFP dimer extracted from the crystal structure appears, therefore, as a reasonable representative system for modeling the early stage of SF in the cocrystals. This system will hence be adopted in the following $a b$ initio and quantum dynamics calculations.

The excitation energies in the PEN:PFP dimer are calculated by the multireference second order perturbation theory ${ }^{36,37}$ with a complete active-space self-consistent field considering four active orbitals [CASSCF $(4,4) / M R M P 2]$. The calculations yield vertical transition energies to $S_{1}$ and TT states, respectively, bracketing from above and below the lowest CT state with the hole localized on the PEN and the electron on PFP, as shown in Table I. The calculated CT state is found to lie at an energy below the PEN and PFP singlet excitons, in accordance with optical absorption measurements. We thus expect singlet fission to be exergonic, irrespective of whether the initial conditions prepare $S_{1}$ or CT excitations. It is worth stressing that it is the lower triplet energy on PFP $(0.65 \mathrm{eV})$, as compared to the PEN T1 $(0.81 \mathrm{eV})$, that plunges the TT pair below the bound CT state. Note that the TT state in PFP:PEN is stabilized from the sum of the T1 energies on isolated PFP and PEN molecules by $\sim 0.03 \mathrm{eV}$. Such a TT stabilization, which is expected to be an activation barrier to generate free triplets from the bound TT pairs, originates from a small CT admixture in the TT wavefunction( 2\%).

The dynamics of SF in a PFP:PEN dimer is simulated by using the multiconfiguration time-dependent Hartree (MCTDH) method. ${ }^{38}$ The model Hamiltonian used in the SF simulations (see Table I) is parameterized based on diabatization of the MRMP2 excited states following a similar protocol as the one used to model

TABLE I. Model Hamiltonian for quantum dynamics calculations of SF in PEN:PFP cocrystals. The excitation energy and coupling $(\mathrm{eV})$ between the states are evaluated using CASSCF(4,4)/MRMP2 calculations. The calculated $\mathrm{S}_{1}$-PEN energy is somewhat underestimated and thus calibrated to reproduce the experimental absorption energy. The intramolecular reorganization energies $(\mathrm{eV})$ of TT, $\mathrm{S}_{1}-\mathrm{PFP}, \mathrm{S}_{1}-\mathrm{PEN}$, and $C T$ are $0.42,0.14,0.14$, and 0.16 , respectively. These are evaluated via geometry optimizations on the respective states using TD-DFT employing the B3LYP functional.

\begin{tabular}{lcccc}
\hline \hline & TT & S $_{1}$-PFP & S $_{1}$-PEN & CT (PFP-:PEN+) \\
\hline TT & \multirow{2}{*}{1.43} & 0.002 & -0.001 & -0.03 \\
S $_{1}$-PFP & & 1.76 & 0.04 & -0.04 \\
S $_{1}$-PEN & & & 1.96 & 0.03 \\
CT (PFP-:PEN+) & & & & 1.57 \\
\hline
\end{tabular}


a)
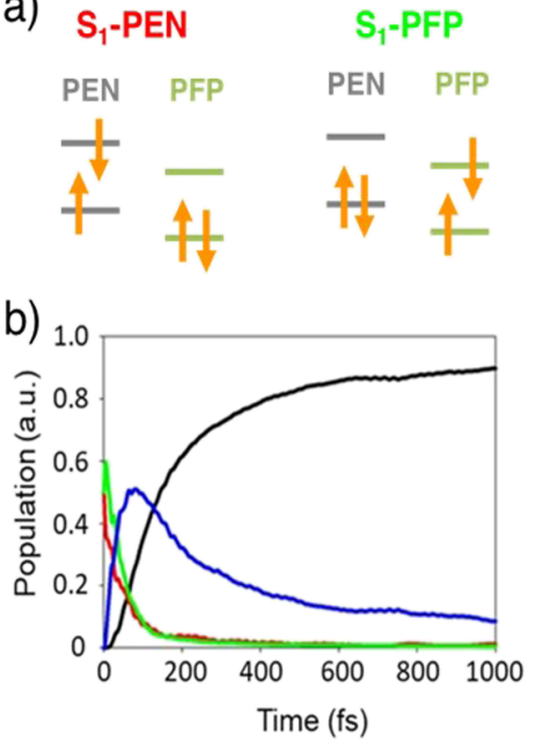
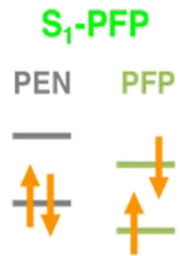

CT

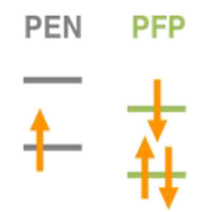

TT

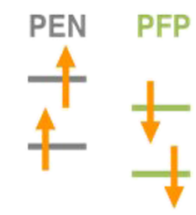

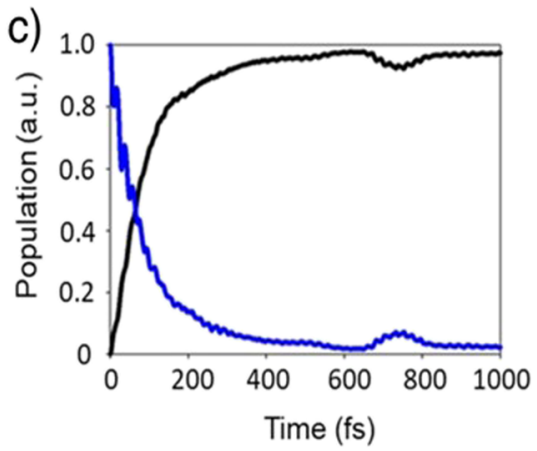

FIG. 5. (a) Sketch of the electronic states involved in the SF process in PEN:PFP cocrystals. [(a) and (b)] Population of TT (black), CT (blue), $S_{1}$ on PEN (red), and $S_{1}$ on PFP (green) during the quantum dynamics calculations for the following initial conditions: (b) equally populated bright singlet excitons of PEN and PFP (c) direct excitation of the $\mathrm{CT}$ state.
SF in acene single crystals. ${ }^{39}$ We consider the four lowest-energy excitations sketched in Fig. 5(a), as obtained from multireference calculations, and 18 intramolecular vibrational modes per molecule, both for PEN and PFP, thus 36 modes in total. The resulting adiabatic states have mixed character, namely, $S_{1}$ now includes a significant fraction of CT configurations ( $6 \%$ according to our CASSCF calculations). Two quantum dynamics simulations have been performed, differing for the initial state and both showing SF in ultrafast time scale. Upon initial excitation of the bright exciton (quantum superposition of the singlet excitons of PEN and PFP molecules), an ultrafast SF proceeds in PFP:PEN over a time scale of $\sim 170 \mathrm{fs}$, in reasonable agreement with the experimental results. Most interestingly, the time-dependent populations reported in Fig. 5(b) show that the $S_{1}$ exciton initially first decays to the intermediate CT state, which then mediates TT formation. In other words, we observe a transient population transfer to CT states after optical excitation into $\mathrm{S}_{1}$. Moreover, in line with the spectroscopic data, direct excitation of CT in PFP:PEN also results in an ultrafast SF, yet on a slightly shorter time scale of $\sim 100$ fs [Fig. 5(c)].

For completeness, we also modeled SF for PFP:PFP and PEN:PEN dimers extracted along the $c$-axis in the PFP:PEN cocrystal. The intermediate CT states in the PFP:PFP and PEN:PEN dimers are energetically higher than the $S_{1}$ states by $\sim 1.1 \mathrm{eV}$, and the direct $\mathrm{S}_{1}$-TT couplings are vanishingly small (see the supplementary material). Hence, not surprisingly, SF is very slow, preventing a quantitative estimate of a time scale from MCTDH simulations. Thus, SF proceeds exclusively along the PFP:PEN dimer, i.e., along the $b$-axis, in the cocrystal. Note that, in PFP:PEN 1:3 films, an ultrafast SF may also occur in the pure PEN regions as observed in PEN single crystals, yet the formation of transient CT states in these domains cannot be unambiguously assessed experimentally.

In conclusion, we have studied the dynamics of mixed PFP:PEN thin films fabricated via molecular beam deposition. The films show charge-transfer states between PFP and PEN in the ground-state absorption. Ultrafast transient absorption measurements combined to nonadiabatic excited-state dynamics simulations show that singlet fission in these films proceeds via a sequential mechanism from singlets to CT states to TT states. Furthermore, we show that fission occurs even when the CT states are directly excited from the ground state. Our results represent, to the best of our knowledge, the first direct observation of CT intermediates for singlet fission in molecular solids.

See the supplementary material for additional transient spectroscopy data and modeling details.

I.S. acknowledges support from the Natural Sciences and Engineering Research Council of Canada (NSERC) (Funding Ref. No. RGPIN-2018-05092) and Concordia University. The authors thank the Winton Programme for the Physics of Sustainability and the Engineering and Physical Sciences Research Council for funding. The work in Mons was supported by Fonds de la Recherche Scientifique de Belgique (FNRS-F.R.S). Computational resources have been provided by the Consortium des Equipements de Calcul Intensif (CECI), funded by the F.R.S.-FNRS under Grant No. 2.5020.1 and by the Walloon Region. D.B. is FNRS research director.

\section{REFERENCES}

${ }^{1}$ M. B. Smith and J. Michl, Chem. Rev. 110, 6891 (2010).

${ }^{2}$ S. Singh, W. J. Jones, W. Siebrand, B. P. Stoicheff, and W. G. Schneider, J. Chem. Phys, 42, 330 (1965).

${ }^{3}$ C. Swenberg and W. Stacy, Chem. Phys. Lett. 2, 327 (1968).

${ }^{4}$ M. C. Hanna and A. J. Nozik, J. Appl. Phys. 100, 074510 (2006).

${ }^{\mathbf{5}}$ P. J. Jadhav, A. Mohanty, J. Sussman, J. Lee, and M. A. Baldo, Nano Lett. 11, 1495 (2011).

${ }^{6}$ B. Ehrler, M. W. B. Wilson, A. Rao, R. H. Friend, and N. C. Greenham, Nano Lett. 12, 1053 (2012).

${ }^{7}$ B. Ehrler, B. J. Walker, M. L. Bohm, M. W. B. Wilson, Y. Vaynzof, R. H. Friend, and N. C. Greenham, Nat. Commun. 3, 1019 (2012). 
${ }^{8}$ B. Ehrler, K. P. Musselman, M. L. Bohm, R. H. Friend, and N. C. Greenham, Appl. Phys. Lett. 101, 153507 (2012).

${ }^{9}$ W. L. Chan, M. Ligges, A. Jailaubekov, L. Kaake, L. Miaja'Avila, and X.-Y. Zhu, Science 334, 1541 (2011).

${ }^{10}$ T. C. Berkelbach, M. S. Hybertsen, and D. R. Reichman, J. Chem. Phys. 138, 114103 (2013)

${ }^{11}$ J. J. Burdett and C. J. Bardeen, J. Am. Chem. Soc. 134, 8597 (2012).

${ }^{12}$ N. Monahan and X. Y. Zhu, Annu. Rev. Phys. Chem. 66, 601 (2015).

${ }^{13}$ D. Beljonne, H. Yamagata, J. L. Brédas, F. C. Spano, and Y. Olivier, Phys. Rev. Lett. 110, 226402 (2013).

${ }^{14}$ G. D. Scholes, J. Phys. Chem. A 119, 12699 (2015).

${ }^{15}$ E. C. Greyson, J. Vura Weis, J. Michl, and M. A. Ratner, J. Phys. Chem. B 114, $14168(2010)$

${ }^{16}$ A. A. Bakulin, S. E. Morgan, T. B. Kehoe, M. W. B. Wilson, A. W. Chin, D. Zigmantas, D. Egorova, and A. Rao, Nat. Chem. 8, 16 (2016).

${ }^{17}$ J. C. Johnson, A. J. Nozik, and J. Michl, Acc. Chem. Res. 46, 1290 (2013).

${ }^{18}$ B. J. Walker, A. J. Musser, D. Beljonne, and R. H. Friend, Nat. Chem. 5, 1019 (2013).

${ }^{19}$ E. Busby, J. Xia, Q. Wu, J. Z. Low, R. Song, J. R. Miller, X.-Y. Zhu, L. M. Campos, and M. Y. Sfeir, Nat. Mater. 14, 426 (2015).

${ }^{20}$ E. A. Marguiles, C. E. Miller, Y. W. Wu, L. Ma, G. C. Schatz, R. M. Young, and M. R. Wasielewski, Nat. Chem. 8, 1120 (2016).

${ }^{21}$ K. Broch, U. Heinemeyer, A. Hinderhofer, F. Anger, R. Scholz, A. Gerlach, and F. Schreiber, Phys. Rev. B 83, 245307 (2011).

${ }^{22}$ A. Rinn, T. Breuer, J. Wiegand, M. Beck, J. Hubner, R. C. Doring, M. Oestreich, W. Heimbrodt, G. Witte, and S. Chatterjee, ACS Appl. Mater. Interfaces 9, 42020 (2017).

${ }^{23}$ A. Rao, M. W. B. Wilson, J. M. Hodgkiss, S. Albert-Seifried, H. Bässler, and R. H. Friend, J. Am. Chem. Soc. 132, 12698 (2010).
${ }^{24}$ H. Marciniak, M. Fiebig, M. Huth, S. Schiefer, B. Nickel, F. Selmaier, and S. Lochbrunner, Phys. Rev. Lett. 99, 176402 (2007).

${ }^{25}$ V. K. Thorsmølle, R. D. Averitt, J. Demsar, D. L. Smith, S. Tretiak, R. L. Martin, X. Chi, B. K. Crone, A. P. Ramirez, and A. J. Taylor, Phys. Rev. Lett. 102, 017401 (2009).

${ }^{26}$ K. Kolata, T. Breuer, G. Witte, and S. Chatterjee, ACS Nano 8, 7377 (2014).

${ }^{27}$ F. Anger, J. O. Ossó, U. Heinemeyer, K. Broch, R. Scholz, A. Gerlach, and F. Schreiber, J. Chem. Phys. 136, 054701 (2012).

${ }^{28}$ I. Salzmann, S. Duhm, G. Heimel, J. P. Rabe, N. Koch, M. Oehzelt, Y. Sakamoto, and T. Suzuki, Langmuir 24, 7294 (2008).

${ }^{29}$ A. Hinderhofer, C. Frank, T. Hosokai, A. Resta, and A. Gerlach, J. Chem. Phys. 134, 104702 (2011).

${ }^{30}$ G. D’Avino, S. Duhm, R. G. Della Valle, G. Heimel, N. Ueno, D. Beljonne, and I. Salzmann, "Electrostatic interactions shape the molecular organization and the electronic structure of organic semiconductor blends" (unpublished).

${ }^{31}$ J. Simbrunner, S. Hofer, B. Schrode, Y. Garmshausen, S. Hecht, R. Resel, and I. Salzmann, J. Appl. Crystallogr. 52, 428 (2019).

${ }^{32}$ J. Simbrunner, C. Simbrunner, B. Schrode, C. Rothel, N. Bedoya-Martinez, I. Salzmann, and R. Resel, Acta Crystallogr., Sect. A: Found. Adv. 74, 373 (2018).

${ }^{33}$ E. V. Tsiper and Z. G. Soos, Phys. Rev. B 64, 195124 (2001).

${ }^{34}$ G. D'Avino, L. Muccioli, C. Zannoni, D. Beljonne, and Z. G. Soos, J. Chem. Theory Comput. 10, 4959 (2014).

${ }^{35}$ G. D’Avino, L. Muccioli, F. Castet, D. Poelking, D. Andrienko, Z. G. Soos, J. Cornil, and D. Beljonne, J. Phys.: Condens. Matter 28, 433002 (2016).

${ }^{36}$ H. Nakano, J. Chem. Phys. 99, 7983 (1993).

${ }^{37}$ M. W. Schmidt, K. K. Baldridge, J. A. Boatz, S. T. Elbert, M. S. Gordon, J. H. Jensen, S. Koseki, N. Matsunaga, K. A. Nguyen, S. Su et al., J. Comput. Chem. 14, 1347 (1993).

${ }^{38}$ H. D. Meyer, U. Manthe, and L. S. Cederbaum, Chem. Phys. Lett. 165, 73 (1990).

${ }^{39}$ H. Tamura, M. Huix-Rotllant, I. Burghardt, Y. Olivier, and D. Beljonne, Phys. Rev. Lett. 115, 107401 (2015). 